\title{
A Mitogen-Activated Protein Kinase Cascade in the CA1/CA2 Subfield of the Dorsal Hippocampus Is Essential for Long-Term Spatial Memory
}

\author{
Sonja Blum, Anthony N. Moore, Frank Adams, and Pramod K. Dash \\ Department of Neurobiology and Anatomy, W. M. Keck Center for the Neurobiology of Learning and Memory, The \\ University of Texas Medical School, Houston, Texas 77030
}

Behavioral, biophysical, and pharmacological studies have implicated the hippocampus in the formation and storage of spatial memory. However, the molecular mechanisms underlying long-term spatial memory are poorly understood. In this study, we show that mitogen-activated protein kinase (MAPK, also called ERK) is activated in the dorsal, but not the ventral, hippocampus of rats after training in a spatial memory task, the Morris water maze. The activation was expressed as enhanced phosphorylation of MAPK in the pyramidal neurons of the CA1/CA2 subfield. In contrast, no increase in the percentage of phospho-MAPK-positive cells was detected in either the CA3 subfield or the dentate gyrus. The enhanced phosphorylation was observed only after multiple training trials but not after a

Clinical and experimental studies have shown that certain types of memory, including declarative memory in humans and spatial memory in animals, are dependent on the hippocampus and possibly the entorhinal, perirhinal, and parahippocampal cortices (Morris et al., 1982; O’Dell et al., 1992; Squire, 1992; Meunier et al., 1993; Sacktor et al., 1993; Suzuki et al., 1993; Barnes et al., 1994; Nicoll and Malenka, 1995; Bunsey and Eichenbaum, 1996; Lanahan et al., 1997). For example, neuropsychological experiments have revealed that hippocampal lesions severely impair a person's ability to acquire declarative memories (Scoville and Milner, 1957; Zola-Morgan et al., 1986). Likewise, hippocampal lesions and pharmacological interventions in rodents have been shown to cause poor performances in several spatial memory tasks (Morris et al., 1982; Moser et al., 1993, 1995). Based on anatomical differences, the dorsal and ventral hippocampi are thought to serve different functions in memory storage (Amaral and Witter, 1995). For example, the dorsal hippocampus dominates the projections to the perirhinal cortex, whereas only the ventral hippocampus is connected to the amygdala. Selective hippocampal lesions in rodents indicate that the dorsal half is critical for spatial memory (Moser et al., 1995). Moreover, electrophysiological recordings suggest that spatial information is processed in the dorsal, whereas nonspatial information is processed in the ventral, hippocampus (Jung et al., 1994; Colombo et

\footnotetext{
Received Dec. 31, 1998; revised Feb. 12, 1999; accepted Feb. 16, 1999.

This work was supported by Grants MH49662 and NS35457 from the National Institutes of Health. We thank Drs. James Knierim, Terry Crow, and John Byrne for their insightful comments. We also thank Dr. Steve Massey for his assistance in the use of the confocal microscope. In addition, we acknowledge the contributions of Dr. Shi-Jie Liu, Saira Beg, and Jennifer Schmidt for their technical assistance.

Correspondence should be addressed to Dr. Pramod K. Dash at the above address.

Copyright (C) 1999 Society for Neuroscience $\quad 0270-6474 / 99 / 193535-10 \$ 05.00 / 0$
}

single trial or after multiple trials in which the location of the target platform was randomly changed between each trial. Inhibition of the MAPK/ERK cascade in dorsal hippocampi did not impair acquisition, but blocked the formation of long-term spatial memory. In contrast, intrahippocampal infusion of SB203580, a specific inhibitor of the stress-activated MAPK (p38 MAPK), did not interfere with memory storage. These results demonstrate a MAPK-mediated cellular event in the CA1/CA2 subfields of the dorsal hippocampus that is critical for long-term spatial memory.

Key words: MAPK; hippocampus; Morris water maze; longterm memory; PD098059; p38MAPK al., 1998). Although the role of the dorsal hippocampus in spatial memory is beginning to emerge, the biochemical and molecular steps underlying this process are poorly understood.

Anatomical evidence suggests that the hippocampus can be roughly divided into four subfields: dentate gyrus, CA3, CA2, and CA1. The flow of spatial information processing takes place via parallel and series transfer through monosynaptic, disynaptic, and trisynaptic connections to influence CA1 neurons, the primary output of the hippocampus (Amaral and Witter, 1995). The entorhinal cortex sends its multimodal outputs to the dentate gyrus, CA3, and CA1 via the perforant path. Output from the dentate gyrus is conveyed to CA3 neurons via mossy fiber axons, and the CA3 neurons project onto the CA1 neurons via Schaffer collateral/commissural axons. Although the intrahippocampal organization is well documented, little information exists regarding the roles of the individual subfields in memory storage. Recent hippocampal long-term potentiation (LTP; a proposed synaptic mechanism for spatial memory) studies in genetically impaired mice have indicated that plasticity at CA1 synapses, in contrast to dentate gyrus and CA3 synapses, is critical for spatial memory (Tsien et al., 1996; Chen and Tonegawa, 1997). However, no direct evidence indicating the subfields may have disparate roles in memory storage has been reported.

Memory in the hippocampus can last for minutes (short-term) to days (long-term) (Milner et al., 1998). Lesions of the hippocampus (both in rodents and in monkeys) indicate that the role of the hippocampus is greatly diminished after $\sim 4-5$ weeks, and a more permanent store develops in the neocortex (Zola-Morgan and Squire, 1990; Kim and Fanselow, 1992). Unlike short-term memory, the formation of long-term memory is thought to depend on gene expression and protein synthesis (Davis and Squire, 
1984; Goelet et al., 1986). Specifically, gene expression via the $\mathrm{Ca}^{2+} / \mathrm{cAMP}$ response element-binding protein (CREB) has been implicated (Bourtchuladze et al., 1994; Alberini et al., 1995; Guzowski and McGaugh, 1997; Kornhauser and Greenberg, 1997; Abel et al., 1998). However, the biochemical steps underlying long-term spatial memory have not been well established. In this report, we show that behavioral training in the Morris water maze activates mitogen-activated protein kinase (MAPK) in the CA1/CA2 neurons of the dorsal hippocampus. In vivo inhibition of the MAPK/ERK cascade in the dorsal hippocampus did not impair acquisition but blocked long-term memory. These results show that CA1/CA2 neurons of the dorsal hippocampus require activation of a MAPK cascade for the formation of normal long-term, but not short-term, spatial memory. This activation was found only in the dorsal, and not the ventral, hippocampus. In addition, our results suggest a disparity in the roles or biochemical mechanisms used between the subfields of the hippocampus in long-term memory storage.

\section{MATERIALS AND METHODS}

Materials. Long-Evans rats were purchased from Harlan Sprague Dawley (Indianapolis, IN). Phospho-MAPK and MAPK antibodies were obtained from New England Biolabs (Beverly, MA). Anti-active c-Jun $\mathrm{N}$-terminal protein kinase (JNK) antibodies and anti-rabbit HRPconjugated secondary antibodies were purchased from Promega (Madison, WI) and Sigma (St. Louis, MO), respectively. Cy3-conjugated streptavidin was obtained from Jackson Immunochemicals (West Grove, PA), PD098059 was purchased from Biomol (Plymouth Meeting, PA), and SB203580 was purchased from Calbiochem (San Diego, CA).

Intrahippocampal infusion and drug preparation. All protocols involving the use of animals are in compliance with the National Institutes of Health's Guide for the Care and Use of Laboratory Animals and approved by the Institutional Animal Care and Use Committee. Male Long-Evans rats $(250-300 \mathrm{gm})$ were anesthetized with $400 \mathrm{mg} / \mathrm{kg}$ chloral hydrate and implanted with bilateral guide cannulas aimed at the dorsal hippocampus (anteroposterior, $-3.3 \mathrm{~mm}$; lateral, $\pm 2.0 \mathrm{~mm}$ from bregma; and ventral, $-1.5 \mathrm{~mm}$ from the dura). The rats were allowed to recover from surgery in their home cages for 10-12 d. During infusion, the injection cannulas extended $1.5 \mathrm{~mm}$ beyond the tips of the guides, yielding a total depth of $3.0 \mathrm{~mm}$ below the dura. Stock solutions of PD098059 was prepared by dissolving in DMSO. Before infusion, PD098059 was diluted in sterile saline yielding either a $2 \mathrm{mg} / \mathrm{ml}$ solution in $40 \%$ DMSO or a $0.2 \mathrm{mg} / \mathrm{ml}$ solution in $4 \%$ DMSO. SB203580 was prepared by dissolving in DMSO and was diluted in sterile saline to a concentration of $0.75 \mathrm{mg} / \mathrm{ml}$ in $10 \%$ DMSO. Vehicle-treated animals were infused with saline and an amount of DMSO corresponding to that used to dissolve the drug (4 or 40\% DMSO for 0.2 and $2.0 \mu \mathrm{g}$ of PD098059, respectively, and 10\% DMSO for SB203580). All injections (1 $\mu$ l/side of either drug or vehicle) were performed in freely moving animals at a rate of 0.25 $\mu \mathrm{l} / \mathrm{min}$ using a dual syringe infusion pump (Stoelting).

Behavioral training. All behavioral training was performed by an experimenter who was kept blind to the treatment schedule. All training was completed in a single day. For post-training infusion experiments, naive animals were trained in the hidden platform version of the Morris water maze task (Morris et al., 1982, 1986; Dixon et al., 1994) with an intertrial interval (iti) of 4 min until they could locate the platform three consecutive times in $15 \mathrm{sec}$. Animals that failed to reach this criterion by trial 12 were eliminated from the study. Each trial was initiated by placing the animal in one of four randomly chosen locations facing the wall of the tank. Animals were allowed to search for the hidden platform for a period of $60 \mathrm{sec}$. If an animal failed to find the platform, it was placed there by the experimenter. Animals were allowed to remain on the platform for a period of $30 \mathrm{sec}$ before being returned to their home cages. For pretraining infusion experiments, naive animals were given a recovery period of $20 \mathrm{~min}$ in their home cages before the start of behavioral training. The training protocol consisted of 16 trials with an iti of $20 \mathrm{sec}$, as previously described by Guzowski and McGaugh (1997).

Retention testing. All behavioral testing was performed by an experi- menter who was kept blind to the treatment schedule. Animals were tested for retention by placing them in the tank facing the wall at a point opposite the location of the hidden platform, as previously described by Guzowski and McGaugh (1997). Animals were given $60 \mathrm{sec}$ to locate the hidden platform. If an animal failed to find the platform, it was placed there by the experimenter. Animals were allowed to remain on the platform for a period of $30 \mathrm{sec}$ before being returned to their home cages. A $5 \mathrm{~min}$ iti was used between each of the three retention trials. For experiments with SB203580, $0.2 \mu \mathrm{g}$ of PD098059, or delayed infusion of PD098059, retention was assessed by a transfer test in which the hidden platform was removed from the maze, and animals were allowed to search for a period of $60 \mathrm{sec}$. Movement within the maze was monitored using a video camera linked to a tracking software (Chromotrack; San Diego Instruments). The time to platform was calculated as the latency for each animal to cross the site at which the hidden platform was located during training. Using the tracking software, swimming speed was calculated by dividing the cumulative total distance (in centimeters) traversed in each zone by the cumulative dwell time (Hagiwara et al., 1993).

Visible platform task. After the completion of behavioral testing, animals were tested for visual acuity using a visible platform version of the Morris water maze. In this task, the water level was decreased so that the top of the platform was even with the water surface. A large visual cue (abstract sign connected to a metal rod) was attached to the platform to increase its visibility. Animals were initially placed on the platform for 30 sec to familiarize them with the cue. Each rat was given three trials in which they were allowed to search for the platform for a period of $60 \mathrm{sec}$. No animal failed to find the platform during this time period. The rat's starting position remained constant throughout the visible testing, but the platform location was randomized between trials to overcome any residual preference for the previous location of the hidden platform. The latencies to reach the visible platform for the three trials was averaged to obtain each animal's visual acuity score.

Histology. Animals were killed by chloral hydrate $(1 \mathrm{gm} / \mathrm{kg})$ overdose and transcardially perfused with $150 \mathrm{ml}$ heparinized $(0.1 \% \mathrm{v} / \mathrm{v})$ PBS followed by $150 \mathrm{ml}$ PBS containing $4 \%$ paraformaldehyde and $15 \%$ picric acid. Brains were removed and post-fixed in perfusant overnight. Brains were then cryoprotected with $30 \%$ sucrose in fixative solution, briefly dried, embedded in OCT (Myers Laboratory), and sectioned on a cryostat into $40-\mu \mathrm{m}$-thick sections. Every sixth section throughout the hippocampus was mounted on gelatin-subbed slides and stained with cresyl violet. The location of the site of infusion was evaluated and recorded for each animal.

Immunohistochemistry. Animals were trained for phospho-MAPK immunohistochemistry, as described for the post-training infusion experiments. Control animals were given either one training trial or $10 \pm 1$ trials in which the platform was moved to a new random location after each trial. At the appropriate time points, animals were killed, and the hippocampal tissues were quickly removed. Tissues were fixed in ice-cold $4 \%$ paraformaldehyde and $15 \%$ picric acid for at least $16 \mathrm{hr}$ and then cryoprotected with $30 \%$ sucrose in PBS. Tissues were briefly dried to remove any surface liquid and embedded in OCT (Myers Laboratory). Forty-micrometer-thick sections were prepared on a cryostat. Freefloating slices were incubated overnight in $1 \mu \mathrm{g} / \mathrm{ml}$ primary antibodies in PBS containing $2 \%$ bovine serum albumin (BSA) and $2.5 \%$ normal goat serum, as described previously (Moore et al., 1996). The immunoreactivity was visualized using a biotinylated secondary antibody and a streptavidin-Cy3 complex, as recommended by the vendor. For doublelabeling experiments, the binding of a neuron-specific antibody (NeuN; Chemicon, Temecula, CA) was detected using an FITC-conjugated secondary antibody. Sections were mounted on microscope slides and coverslipped under $50 \%$ glycerol in $0.25 \mathrm{M}$ Tris- $\mathrm{HCl}, \mathrm{pH} 9.5$. Fluorescence was protected using paraphenylenediamine $(1 \mathrm{mg} / \mathrm{ml}$ in mounting media) and observed using a UV microscope with the appropriate filter set (Axiophot). The number of immunopositive cells for each animal was calculated as an average of three randomly chosen $1350 \times 900 \mu \mathrm{m}$ areas from three different slices counted by two independent blind observers. The total number of cells in these areas was counted after staining sections with cresyl violet. The results for each group were obtained from five animals.

Confocal microscopy. Nuclear signal for phospho-MAPK was assessed using a Zeiss krypton-argon laser confocal microscope. Digitized images were captured by a computer using Zeiss LSM software. Nuclear immunoreactivity was quantified by comparing the average pixel intensity of a defined area within the nucleus with a size-matched area of the 
cytoplasm for each individual neuron. The data were compiled from five randomly chosen fields.

Preparation of protein extracts. At the appropriate time points, animals were killed, and the hippocampal tissues were quickly removed as described in the immunohistochemistry section. The tissues were homogenized in 10 volumes of $10 \mathrm{~mm}$ Tris-HCl, pH 7.4, $1 \mathrm{~mm}$ EGTA, $1 \mathrm{~mm}$ EDTA, $0.5 \mathrm{~mm}$ DTT, $0.5 \mathrm{~mm}$ PMSF, $10 \mu \mathrm{g} / \mathrm{ml}$ leupeptin, and phosphatase inhibitors $\left(2 \mathrm{~mm} \mathrm{NaF}, 2 \mathrm{~mm} \mathrm{NaPP}_{i}, 5 \mu \mathrm{M}\right.$ microcystin-LR, and $1 \mathrm{~mm}$ $\mathrm{Na}_{3} \mathrm{VO}_{4}$ ). After 20 strokes in a motor-driven Teflon-glass homogenizer, a portion of each sample was aliquoted and immediately frozen at $-80^{\circ} \mathrm{C}$. The rest of the homogenate was aliquoted and centrifuged at $15,000 \times g$ for $30 \mathrm{~min}$ at $4^{\circ} \mathrm{C}$. The pelletized material and supernatant solutions were separated and stored at $-80^{\circ} \mathrm{C}$ until needed.

Western blots. The amount of protein in each sample was measured using a MicroBCA assay (Pierce, Rockford, IL) using BSA as the standard. Samples were prepared by boiling in sample buffer, and equal amounts of protein were resolved in a $10 \%$ Tris-Tricine SDS-PAGE gel and transferred to an Immobilon-P (Millipore, Bedford, MA) membrane using a semidry transfer apparatus (Millipore). Membranes were blocked overnight in $5 \%$ BSA in PBS and incubated with $0.2 \mu \mathrm{g} / \mathrm{ml}$ the primary antibodies for $3 \mathrm{hr}$ at room temperature. Membranes were then given five $10 \mathrm{~min}$ washes in TBST (10 mM Tris-HCl, pH 7.9, $150 \mathrm{~mm} \mathrm{NaCl}$, and $0.05 \%$ Tween 20). Immunoreactivity was assessed by a HRP-conjugated secondary antibody and chemiluminescence, which allowed the production of multiple fluorographs with different lengths of exposure. The quantification of the immunoreactive bands was performed using a Bio-Rad (Hercules, CA) model GS-670 imaging densitometer. The data represents the average of four independent experiments. Before reprobing, blots were stripped by two $10 \mathrm{~min}$ washes in a buffer containing 62 $\mathrm{mm}$ Tris- $\mathrm{HCl}, \mathrm{pH} 6.8,2 \% \mathrm{SDS}$, and $100 \mathrm{~mm} \beta$-mercaptoethanol at $50^{\circ} \mathrm{C}$. The membranes were then washed extensively with TBST and reblocked overnight in $2 \%$ BSA.

Kinase assays. Calcium-calmodulin-dependent protein kinase (CaMK) activity was measured using a synthetic peptide substrate (autocamtide-3; KKALHRQETVDAL), as described by Moore et al. (1996). Phosphorylation reactions were initiated by adding $3 \mu \mathrm{g}$ of protein to a $20 \mu \mathrm{l}$ mixture, resulting in the following final concentrations: $10 \mathrm{~mm}$ HEPES, pH 7.4, $0.5 \mathrm{~mm}$ DTT, $20 \mu \mathrm{M}$ substrate peptide, $50 \mu \mathrm{M}$ ATP, $2.0 \mathrm{mM} \mathrm{CaCl} 2,2 \mu \mathrm{M}$ calmodulin, $5 \mathrm{mM} \mathrm{MgCl}_{2}$, and $2 \mu \mathrm{Ci}$ of $\left[\gamma^{-32} \mathrm{P}\right]$ ATP $(3000 \mathrm{Ci} / \mathrm{mmol})$. Control reactions for background determination were prepared simultaneously by omitting the substrate peptide. After a $1 \mathrm{~min}$ incubation at $30^{\circ} \mathrm{C}$, the reactions were terminated by spotting $15 \mu \mathrm{l}$ of the reaction mixture on $\mathrm{P}-81$ phosphocellulose filters. The filters were washed three times, $10 \mathrm{~min}$ each, in $75 \mathrm{~mm}$ phosphoric acid, rinsed with ethanol, and dried under air flow. The radioactivity on the phosphorylated peptide substrate was quantitated in a scintillation counter by the Cerenkov method.

cAMP-dependent protein kinase A (PKA) assays using Kemptide (LRRASLG) as a substrate were performed as described previously (Roberson and Sweatt, 1996). The assay mixture consisted of $37.5 \mathrm{~mm}$ MES, pH 6.0, $15 \mathrm{mM} \mathrm{Mg}$ acetate, $30 \mu \mathrm{M}$ ATP, $2 \mu \mathrm{Ci}$ of $\left[\gamma_{-}{ }^{32} \mathrm{P}\right]$ ATP $(3000$ $\mathrm{Ci} / \mathrm{mmol}), 0.2 \mathrm{mM}$ IBMX, $20 \mu \mathrm{M}$ chlorophenylthiol cAMP, and $300 \mu \mathrm{M}$ Kemptide. The phosphorylation reaction was initiated by adding $5 \mu \mathrm{g}$ of protein. Control reactions to determine the background phosphorylation were performed simultaneously by omitting the substrate peptide. The reactions were performed at $30^{\circ} \mathrm{C}$ for $5 \mathrm{~min}$ and terminated by spotting $15 \mu \mathrm{l}$ of reaction mixture on P81 phosphocellulose filters as described above.

Protein kinase $\mathrm{C}$ (PKC) activity was measured using the specific substrate peptide (AAAKIQASFRGHMAR) as described previously (Klann et al., 1993). The assay mixture consisted of $20 \mathrm{mM}$ HEPES, pH 7.4, $10 \mathrm{~mm} \mathrm{MgCl}_{2}, 2 \mathrm{~mm} \mathrm{NaPPi}, 0.1 \mathrm{~mm}$ PMSF, $100 \mu \mathrm{M} \mathrm{ATP,} 1 \mathrm{~mm}$ $\mathrm{CaCl}_{2}, 320 \mu \mathrm{g} / \mathrm{ml}$ phosphatidylserine, $30 \mu \mathrm{g} / \mathrm{ml}$ diacylglycerol, $2 \mu \mathrm{Ci}$ of $\left[\gamma-{ }^{32} \mathrm{P}\right] \mathrm{ATP}$, and $20 \mu \mathrm{M}$ substrate peptide. The reaction mixture was incubated with $3 \mu \mathrm{g}$ of protein extract for $1 \mathrm{~min}$ at $30^{\circ} \mathrm{C}$. The reactions were spotted on P81 paper and processed as described above.

Statistical analysis. Statistical significance was determined by an ANOVA followed by appropriate post hoc analysis or by a two-tailed Student's $t$ test for unpaired variables. Data were considered significant at $p \leq 0.05$. Statistical analyses were performed using either the integrated optical densities (Western blots), cell counts (as percentage of total cells for immunohistochemistry), counts per minute per milligram of protein (kinase assays), or latencies (behavior).

\section{RESULTS \\ MAPK is activated in the dorsal hippocampus after behavioral training}

MAPK is activated when phosphorylated on $\mathrm{Thr}^{202}$ and $\mathrm{Tyr}^{204}$ residues by the upstream kinase MAPKK (or MEK). An antibody that specifically recognizes dually phosphorylated MAPK (both p42 and p44 isoforms) as well as an antibody against total MAPK was used to examine its activation and protein levels in the hippocampus after training of rats in the Morris water maze. Animals were trained to find the location of a hidden platform in $<15 \mathrm{sec}$, three consecutive times. This training protocol reproducibly generates a long-term memory for the location of the hidden platform. After the completion of the last training trial, hippocampi were removed, divided into dorsal and ventral halves, and used for Western blots and immunohistochemistry. Western blot analysis did not reveal any changes in either the phosphorylation or the total levels of MAPK (data not shown). If MAPK phosphorylation is restricted to only a limited population of cells as a result of behavioral training, this change could be masked because of inclusion of large numbers and types of cells in the protein extracts. To circumvent this possibility, immunohistochemistry was used. Figure 1 shows that, compared with naive rats (Fig. 1a), the number of cells immunopositive for phosphorylated MAPK increased by $5 \mathrm{~min}$ after behavioral training in the CA1/CA2 subfields of the dorsal hippocampus (Fig. 1b). The phospho-MAPK immunoreactivity was localized to neurons as determined by double-labeling with the neuron-specific antibody NeuN (Fig. 1c). All phospho-MAPK-immunoreactive cells were also immunopositive for NeuN. No change in the number of phospho-MAPK-immunopositive cells was observed in the CA3 subfield or the dentate gyrus. Control and experimental animals showed comparable total MAPK immunoreactivity in the CA1/ CA2 (Fig. 1d,e) and CA3 subfields, and the dentate gyrus. When the primary antibodies were eliminated from the incubation mixture, no specific immunoreactivity was detected.

Figure $1 f$ summarizes data indicating that animals trained to criterion and killed $5 \mathrm{~min}$ after training had a significantly higher percentage of immunopositive cells (naive, $5.3 \pm 0.8 \%$; trained, $11.5 \pm 1.5 \% ; p<0.05)$ than naives $(n=5)$. Immunopositive cells were counted by two independent blind observers and scored as outlined in Materials and Methods. This increase in immunoreactivity returned to control levels by $30 \mathrm{~min}$ after training. As a control, phospho-MAPK immunohistochemistry was performed in animals that were given a single training trial and killed $5 \mathrm{~min}$ later. Multiple training trials in the Morris water maze task are needed to acquire long-term memory for this task (Morris et al., 1982, 1986). Figure $1 e$ shows that a single training trial does not significantly change the percentage of phospho-MAPK-positive cells compared with naives, indicating that the increased MAPK phosphorylation is not a result of stress or physical activity. As an additional control, an identical protocol to that used for training was used, except that the hidden platform was moved to a randomly chosen new location after each trial to limit the spatial learning component of the task. In this task, animals abandoned thigmotaxis as their primary search strategy, but their latencies to the platform did not improve over the course of training. When examined for phospho-MAPK-positive cells ( $5 \mathrm{~min}$ after the last trial), no significant difference was detected between this group and naive animals. However, both control groups (one trial, randomized platform) were significantly different when compared with animals trained to criterion and killed 5 min later (Fig. $1 f$ ). 

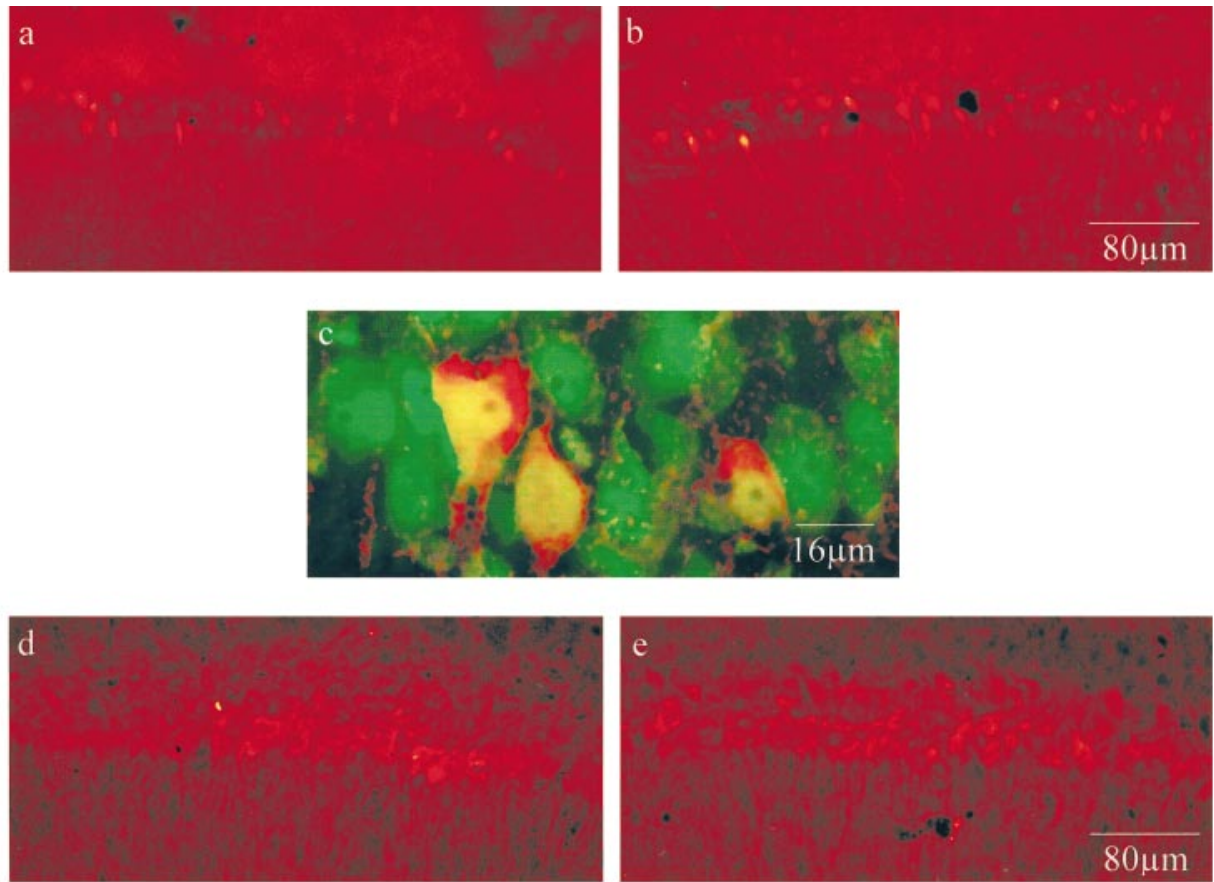

Figure 1. Behavioral training increase MAPK phosphorylation in the CA1/CA2 subfields of the dorsal hippocampus. Representative photomicrographs for phosphoMAPK immunoreactivity in the CA1/CA2 subfields of dorsal hippocampi $(\sim 1.0 \mathrm{~mm}$ from the dorsal tip) from naive $(a)$ and 5 min post-training $(b)$ animals. Training to criterion increases the number of immunopositive cells for phospho-MAPK as compared with naive controls. $c$, Laser confocal image, indicating that the phosphoMAPK-positive cells (red) are also immunoreactive for a neuron-specific nuclear antigen ( green). Areas with overlapping immunofluorescences appear yellow. Representative photomicrographs for MAPK immunoreactivity from a naive $(d)$ and a 5 min post-training (e) animal. $f$, Summary showing percentage of neurons staining positive for phosphorylated MAPK in the dorsal hippocampus from the control and experimental groups. The data are represented as the mean $\pm \operatorname{SEM}(n=5$ for each group). $g$, Representative laser confocal images from a randomized platform and an animal trained to criterion and killed $5 \mathrm{~min}$ later, showing increased numbers of CA1/ CA2 pyramidal neurons with nuclear staining for phospho-MAPK as a result of training. The open and closed arrows indicate neurons with weak or strong phosphoMAPK nuclear immunoreactivity, respectively. The bar graph shows the nuclear to cytoplasmic ratio for phospho-MAPK immunofluroscence. ${ }^{*} p \leq 0.05$; p.t.c., posttraining to criterion.

f
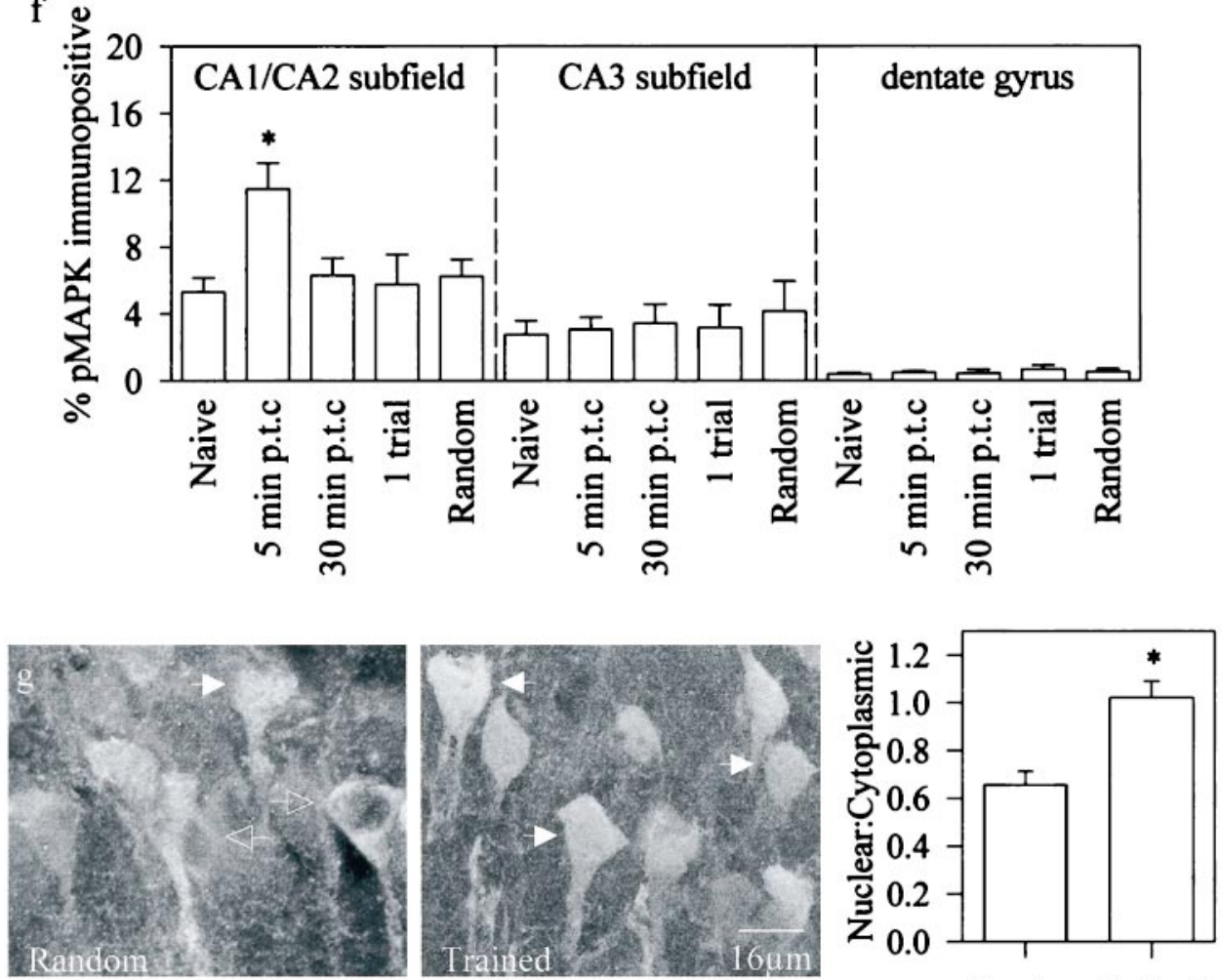

Random Trained

When the phospho-MAPK-immunostained sections were viewed using confocal microscopy, it was observed that animals trained to criterion and killed 5 min later had more cells with nuclear immunoreactivity compared with randomized platform (Fig. 1g) controls. Analysis of nuclear to cytoplasmic signal ratios suggested translocation of MAPK into the nucleus as a result of behavioral training (random, $0.65 \pm 0.05$; trained, $1.02 \pm 0.07$; $p \leq 0.05$ ) (Fig. $1 g$ ). This is consistent with previous in vitro studies that reported that repeated exposure of cells to neurotransmitters or trophic factors causes translocation of MAPK into the nucleus, where it is thought to activate gene expression (Marshall, 1995; Martin et al., 1997).

\section{Behavioral training does not alter MAPK activation in the ventral hippocampus}

In contrast to the dorsal hippocampus, the number of phosphoMAPK-immunopositive neurons in the CA1/CA2 subfields of the ventral hippocampus of animals trained to criterion (Fig. 2b) did 

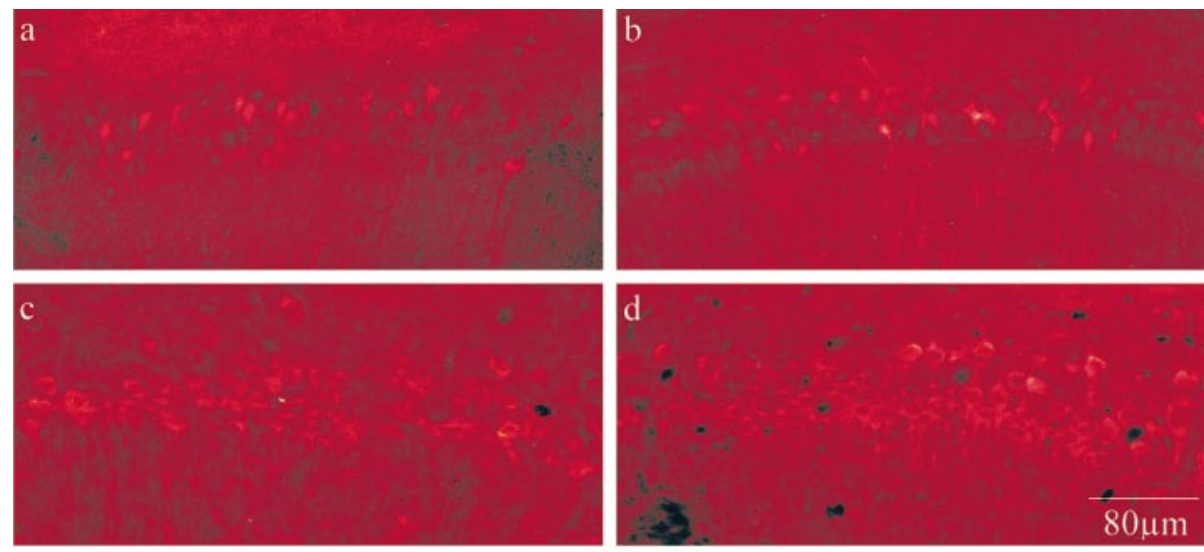

e

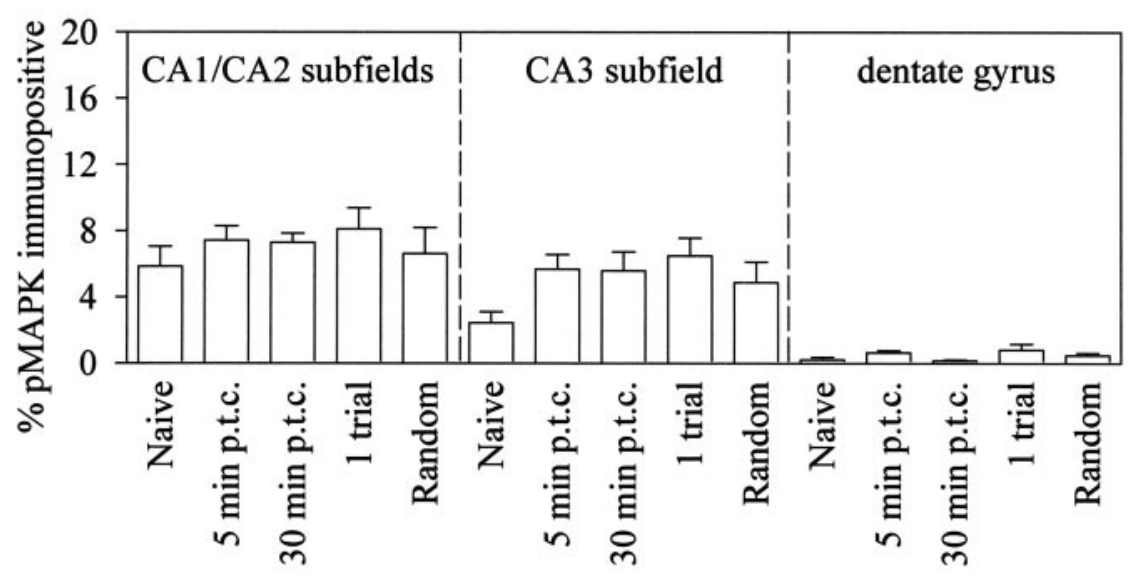

Figure 2. Behavioral training does not increase the percentage of phospho-MAPKimmunopositive cells in the ventral hippocampus. Representative photomicrographs of phospho-MAPK immunoreactivity in the CA1/CA2 subfields of ventral hippocampi $(\sim 1.5 \mathrm{~mm}$ from the ventral tip) from naive $(a)$ and 5 min post-training $(b)$ animals. Representative photomicrographs for MAPK immunoreactivity in adjacent slices from naive $(c)$ and 5 min-trained $(d)$ animals. $e$, Summary figure showing the percentage of phospho-MAPK-immunopositive neurons in the CA1/CA2, CA3, and dentate gyrus subfields. The data are represented as the mean $\pm \operatorname{SEM}(n=5$ for each group). p.t.c. Post-training to criterion. not change compared with naive animals (Fig. 2a). Control and experimental animals showed comparable MAPK immunoreactivity in the CA1/CA2 (Fig. $2 c, d$, respectively) and CA3 subfields, and the dentate gyrus of the ventral hippocampus. Figure $2 e$ shows that the percentage of phospho-MAPK-immunopositive cells in the CA1/CA2 subfield, CA3 subfield, and dentate gyrus did not change as a result of behavioral training. These biochemical findings support previous lesion studies and extend the notion that the dorsal, but not the ventral, hippocampus is critical for spatial memory storage (Moser et al., 1993, 1995; Amaral and Witter, 1995; Colombo et al., 1998).

\section{PD098059 specifically inhibits MAPK phosphorylation}

To further examine the role of MAPK activation in spatial memory, the inhibitor PD098059 was used (Alessi et al., 1995). Before behavioral studies, the specificity of the inhibitor was examined. PD098059 (2 $\mu \mathrm{g}$ ) was infused into one while an equal volume of the vehicle was simultaneously infused into the other dorsal hippocampus of the same animal. Animals were killed at 30 min after injection, and the phosphorylation levels of MAPK were examined in the dorsal hippocampus using Western blots. Figure $3 a$ shows that PD098059 caused an $\sim 35 \%$ reduction in MAPK phosphorylation when compared with the vehicle-treated controls $(n=4)$. When the blots were stripped and reprobed using a MAPK antibody, no change in the total level of MAPK was detected between PD098059- and vehicle-treated samples (Fig. $3 a$ ). Using the same 30 min postinfusion protein extracts, we examined if PD098059 interfered with the activities of other protein kinases. Figure $3 b$ shows that PD098059 injection does not alter the phosphorylation of stress-activated protein kinase
(SAPK) isoforms, related MAPK family members. In addition, the second messenger-stimulated activities of CaMK, PKC, and cAMP-dependent PKA were unchanged as a result of PD098059 infusion (Klann et al., 1993; Moore et al., 1996). These results indicate that the dosage of drug used selectively inhibits the MAPK cascade. When the above infusion protocol was used and animals were trained 20 min later, sections from the drug-infused hippocampus had markedly fewer phospho-MAPK-immunopositive neurons in the CA1/CA2 subfield compared with the vehicleinf used contralateral side (Fig. 3c).

\section{PD098059 infusion blocks long-term spatial memory}

To examine the causal role of MAPK activation in spatial memory, groups of animals were bilaterally infused with either the vehicle or the inhibitor $(2 \mu \mathrm{g}) 20 \mathrm{~min}$ before training in the water maze. Because phospho-MAPK immunoreactivity returned to control levels by $1 \mathrm{hr}$ after the infusion of the inhibitor (data not shown), a 16 trial, $20 \mathrm{sec}$ iti protocol was used to complete the training procedure in $<20 \mathrm{~min}$, as previously described by Guzowski and McGaugh (1997). Animals that completed the last three trials with an average time $<30 \mathrm{sec}$ (failures: vehicle $=6$; PD098059 = 6) were used for this study. Figure $4 a$ shows that there was no significant difference in performance during training of rats treated with vehicle and PD098059, indicating that the MEK inhibitor does not interfere with acquisition. When tested for retention $48 \mathrm{hr}$ after the completion of training, animals treated with PD098059 took significantly longer to find the hidden platform than vehicle-treated controls, indicating impairment of long-term memory (vehicle, $29.59 \pm 9.8 \mathrm{sec}$; PD098059, $52.68 \pm$ $5.2 \mathrm{sec} ; p \leq 0.05)$. Analysis of the swimming speeds for each 
Figure 3. Infusion of PD098059 selectively decreases the phosphorylation of MAPK. $a$, Representative Western blots showing that infusion of PD098059 into the dorsal hippocampus decreases the phosphorylation, but not the total levels, of MAPK in the dorsal hippocampus when examined 30 min after infusion. $b$, Summary table showing that PD098059 inhibits MAPK but not SAPK phosphorylation in the dorsal hippocampus. Moreover, PD098059 infusion does not affect CaMK, PKC, or PKA activities $(n=4) .{ }^{*} p \leq 0.05$ compared with vehicle controls. $c$, Representative photomicrographs showing decreased phospho-MAPK immunoreactivity of CA1/CA2 neurons in the dorsal hippocampus after PD098059 infusion as compared with the vehicle-infused contralateral side.

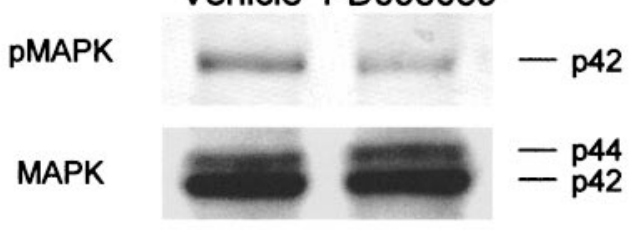

b

Experiment
p42 phosphoMAPK level
p44 phosphoMAPK level
p42 MAPK level
p44 MAPK level
p50 phosphoSAPK level
p52 phosphoSAPK level
CaMK activity
PKC activity
PKA activity

c Vehicle

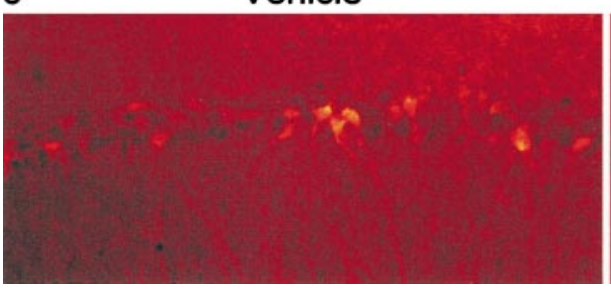

Figure 4. Inhibition of the MAPK cascade attenuates long-term retention in the Morris water maze task. $a$, Bilateral infusion of $2 \mu \mathrm{g} /$ side PD098059 into the dorsal hippocampi $20 \mathrm{~min}$ before training does not interfere with acquisition but attenuates long-term retention (vehicle, $n=6$; PD098059, $n=7)$. Note that the training sessions are plotted as blocks of four trials, whereas the retention trials are plotted individually. The inhibitor did not interfere with the subsequent reacquisition of the task, indicating that the drug does not interfere with visual acuity or motivational state. Representative traces from the first retention trial are shown. $b$, Posttraining bilateral infusion of $2 \mu \mathrm{g} / \mathrm{side}$ PD098059 into dorsal hippocampi attenuates performance in the Morris water maze task when tested $48 \mathrm{hr}$ later (vehicle, $n=12$; PD098059, $n=13$ ). $c$, Posttraining infusion of $0.75 \mu \mathrm{g} / \mathrm{side}$ SB203580 (vehicle, $n=8$; SB203580, $n=$ 8 ) does not interfere with long-term memory. a

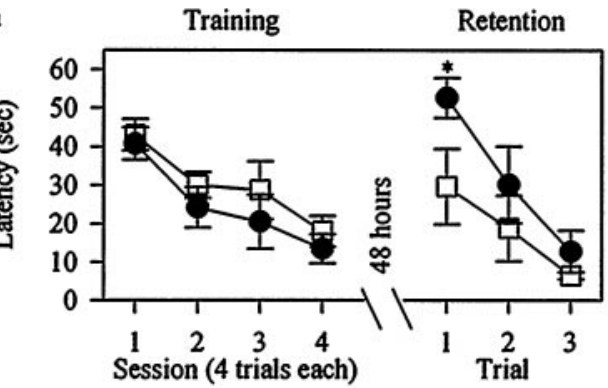

b

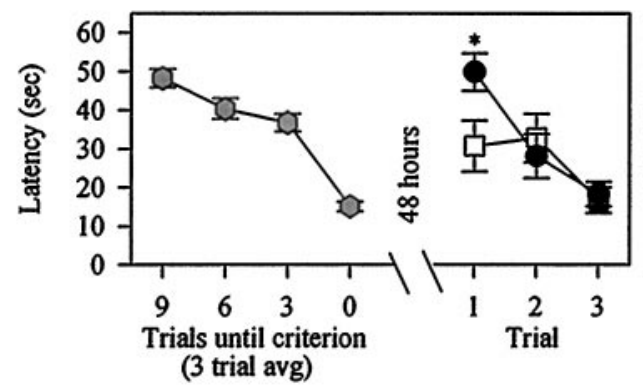

$\%$ Control

$64.87 \pm 6.12 *$

undetectable

$96.15 \pm 4.32$

$94.75 \pm 1.82$

$104.23 \pm 1.97$

$107.50+5.92$

$100.77 \pm 0.24$

$98.60 \pm 8.27$

$108.69 \pm 8.03$

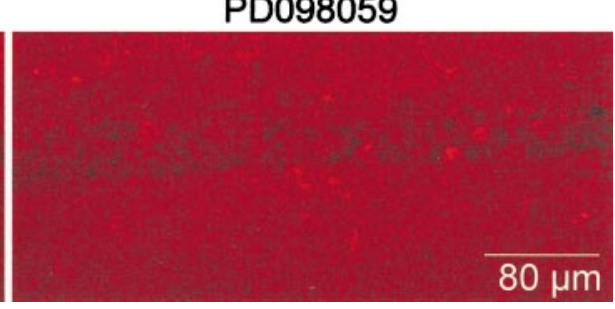

c

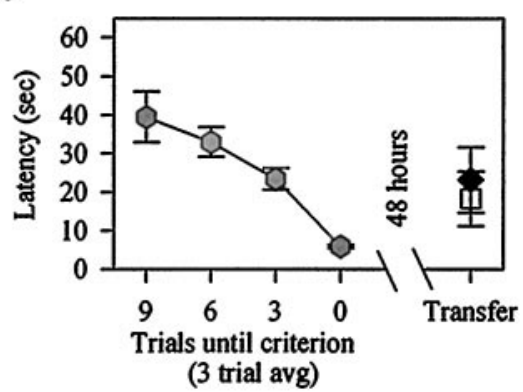

before infusion

vehicle

$2 \mu \mathrm{g}$ PD098059

$0.75 \mu \mathrm{g}$ SB2 203580 
group did not reveal any significant differences (vehicle, $24.82 \pm$ $1.55 \mathrm{~cm} / \mathrm{sec}$; PD098059, $23.5 \pm 1.25 \mathrm{~cm} / \mathrm{sec}$; NS). Representative swim paths for a vehicle and a PD098059-infused animal during the first trial of the retention test are shown. The swim paths for the PD098059-infused animals were similar to those obtained during their first training trial. After retraining, the performance of both groups improved, indicating that PD098059 does not interfere with visual acuity or motivational states and revealed that the experimental group exhibited "savings."

\section{Post-training infusion of PD098059 blocks long-term spatial memory}

The increase in percentage of phospho-MAPK-immunopositive neurons after behavioral training lasts for $<30 \mathrm{~min}$ (Fig. $1 f$ ). To investigate if infusion of PD098059 immediately after training affects spatial memory performance, animals were trained to criterion as described for phospho-MAPK immunohistochemistry. Immediately after the completion of training, animals were randomly divided into two groups and bilaterally infused with either the vehicle or PD098059 $(2 \mu \mathrm{g})$. Figure $4 b$ shows that during the retention test, rats treated with PD098059 took significantly longer to find the platform than those treated with vehicle (vehicle, $30.73 \pm 6.6 \mathrm{sec}$; PD098059, $49.88 \pm 4.85 \mathrm{sec} ; p \leq 0.05$ ). As observed in the preinjection experiment, the performance of PD098059-treated animals on subsequent retraining trials was similar to that of the vehicle-treated group. In contrast to PD098059, post-training infusion of SB203580 $(0.75 \mu \mathrm{g})$, a selective inhibitor of the p38MAPK cascade, did not interfere with long-term spatial memory vehicle (vehicle, $18.31 \pm 7.1 \mathrm{sec}$; SB203580, $23.14 \pm 8.5 \mathrm{sec}$; NS) (Fig. 4c).

The time course for activation of MAPK after behavioral training (Fig. 1f) suggests that PD098059 efficacy may have a time window and that delayed infusion should not interfere with spatial memory. To evaluate this possibility, the infusion of PD098059 was delayed by an hour after the completion of training. Delayed infusion of PD098059 did not significantly alter the performance of animals compared with similarly inf used vehicletreated controls when tested $48 \mathrm{hr}$ after training (Fig. 5a). This finding also shows that the inhibitor does not have any lingering effects on performance.

It was estimated that $2 \mu \mathrm{g}$ of PD098059 (dissolved in 40\% DMSO) infused into one hippocampus resulted in an equilibrium concentration of $\sim 37.5 \mu \mathrm{M}$, although the concentration was likely to be higher immediately surrounding the inf usion site. Previous in vitro studies have used PD098059 concentrations ranging from 10-100 $\mu \mathrm{M}$ (Alessi et al., 1995). To examine the efficacy of a 10-fold lower concentration of PD098059 on spatial memory, 0.2 $\mu \mathrm{g}$ of PD098059 (dissolved in 4\% DMSO) was infused. This concentration of drug decreased MAPK phosphorylation in the dorsal hippocampus when compared with a simultaneously infused vehicle control. Figure $5 b$ shows that bilateral infusion of $0.2 \mu \mathrm{g}$ of PD098059 immediately after training significantly impaired performance in a retention test $48 \mathrm{hr}$ later (vehicle, $17.18 \pm 5.4 \mathrm{sec}$; PD098059, $39.73 \pm 7.2 \mathrm{sec} ; p \leq 0.05)$. When tested using a visible platform version of the Morris water maze, no statistically significant differences in the latency to locate the visible platform was detected between the two groups (vehicle, $9.21 \pm 1.7 \mathrm{sec} ; 0.2 \mu \mathrm{g}$ of PD098059, $8.17 \pm 1.6 \mathrm{sec}$; NS). After the completion of all the behavioral studies, animals were anesthetized, perfused, and brain sections were stained with cresyl violet to determine the locations of the infusion sites. None of the infusion sites were outside the boundaries of the hippocampus

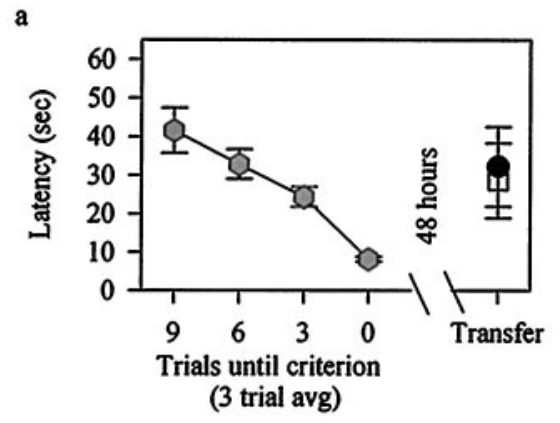

b

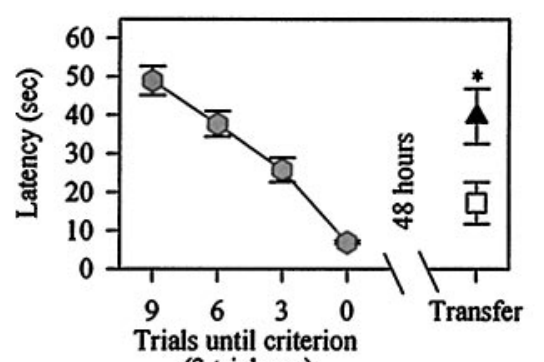

(3 trial avg)
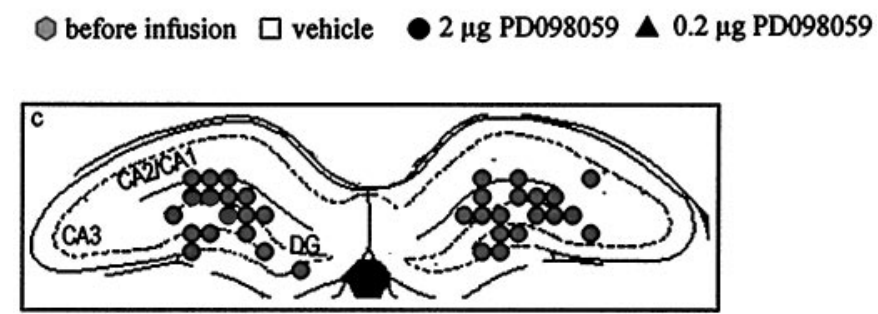

Figure 5. $a$, Delayed ( $1 \mathrm{hr}$ ) infusion of $2 \mu \mathrm{g} /$ side PD098059 does not interfere with long-term memory retention (vehicle, $n=6$; PD098059, $n=6)$. $b$, Post-training bilateral infusion of $0.2 \mu \mathrm{g} /$ side of PD098059 significantly attenuated performance $48 \mathrm{hr}$ later as compared with vehicle (4\% DMSO)-treated animals (vehicle, $n=8$; PD098059, $n=8$ ). $c$, Drawing of a coronal section of the hippocampus indicating sites (gray circles) of infusion for animals used in behavioral studies. Each circle represents a novel infusion site and may represent more than one animal. No sites were outside the boundary of the hippocampus.

(Fig. 5c). No animals were excluded from behavioral analysis based on histology.

\section{DISCUSSION}

The results presented in this report revealed three novel findings relevant to spatial memory. First, behavioral training in a spatial memory task is accompanied by increased number of phosphoMAPK-positive cells in the CA1/CA2 subfield, but not the dentate gyrus or CA3 subfield, of the hippocampus. Second, this enhanced phosphorylation of MAPK after training was restricted to the dorsal hippocampus. Finally, in vivo inhibition of MAPK phosphorylation impairs long-term, but not short-term, spatial memory. These findings demonstrate a critical role for a MAPKmediated cellular event in the CA1/CA2 subfield of the dorsal hippocampus in long-term spatial memory.

Immunohistochemistry for phospho-MAPK indicated that animals not exposed to the Morris water maze show MAPK phosphorylation in $\sim 5 \%$ of neurons in the CA1/CA2 subfield. Control groups (one trial or randomized platform) did not present any increase in the percentage of phospho-MAPK-immunopositive neurons. After training, however, this number increased to $\sim 12 \%$ 
(Fig. 1f), indicating that MAPK activation is specific to the learning component of the task. The observation that increased MAPK phosphorylation is restricted to only CA1/CA2 neurons after behavioral training raises the hypothesis that this subfield is of critical importance in long-term spatial memory storage. This hypothesis is further supported by previous studies that correlated spatial memory with LTP in the various hippocampal subfields using genetically modified mice (Tsien et al., 1996; Chen and Tonegawa, 1997). It has been reported that mice with impaired CA1 LTP, but not mice lacking CA3 or dentate gyrus LTP, show spatial memory deficits (Huang et al., 1995; NostenBertrand et al., 1996). Taken together, our finding of a trainingassociated activation of MAPK in only the CA1/CA2 neurons, combined with the correlation of LTP and spatial memory in this subfield, provides evidence that the different subfields may be serving different functions during long-term memory storage. In addition, based on the proposed role of MAPK in growth and differentiation, this suggests that the CA1/CA2 subfield in particular may be the site for hippocampal plasticity associated with long-term spatial memory.

Previous lesion studies, as well as electrophysiological studies, have indicated that the dorsal hippocampus, in contrast to the ventral hippocampus, may participate in the storage of spatial memory (Jung et al., 1994; Moser et al., 1995; Colombo et al., 1998). For example, Moser et al. (1993) have reported that selective lesions of the dorsal hippocampus impair performance in the Morris water maze task. In contrast, lesion of the ventral side did not produce any observable deficits. Consistent with the proposed role of the dorsal hippocampus in the Morris water maze task, the enhanced MAPK phosphorylation we observed was restricted to only this segment. This increase in MAPK activation was found only after training of animals to criterion and not after training in which the spatial learning component was eliminated (Fig. 1f). "Place cells", cells whose activities correlate with the position of the animal, are believed to play a fundamental role in spatial navigation and are more common in the dorsal hippocampus for both rodents and monkeys (Jung et al., 1994; Colombo et al., 1998). Neuronal activity in response to nonspatial stimuli is found to be evenly distributed throughout the hippocampus. It is not known at present if the neurons that are positive for phosphorylated MAPK are place cells.

To establish a causal role for MAPK activity in spatial memory, we infused PD098059 and assessed its affect on behavioral performance. This inhibitor was found to decrease the phosphorylation of MAPK when compared with simultaneously infused vehicle controls. However, the phosphorylation of a related MAPK family member, SAPK (or JNK), was unaffected. Moreover, the activities of protein kinase A, calcium-calmodulindependent protein kinase and protein kinase $\mathrm{C}$ were also unchanged as a result of PD098059 infusion (Fig. $3 b$ ). However, this study cannot rule out the possibility that PD098059 may be nonspecifically inhibiting other kinases that were not directly examined or that diffusion of the inhibitor during homogenization reduced its efficacy. When PD098059 was infused before behavioral training, immunohistochemical analysis showed a decrease in the number of phospho-MAPK-immunoreactive cells (Fig. 3c). This infusion protocol did not affect acquisition as compared with vehicle-infused animals (Fig. 4a). This indicates that MAPK may not play a role in either hippocampus-dependent learning or short-term memory. However, long-term spatial memory was found to be significantly impaired in the PD098059infused animals when tested $48 \mathrm{hr}$ after training (Fig. $4 a$ ). This difference in performance was not caused by any deficits in motor function or visual acuity because their performance in subsequent retraining trials was not significantly different from the vehicle-inf used controls.

The involvement of MAPK activity in long-term memory was further substantiated by the post-training infusion of PD098059. As predicted by the time course for MAPK activation, immediate infusion, but not delayed infusion, of PD098059 after behavioral training blocked long-term memory. Our data indicates that there is no increase in the number of phospho-MAPK-immunopositive cells after a single training trial. Only multiple training trials resulted in increased number of phospho-MAPK-immunopositive cells. This suggests that MAPK activity, as well as its translocation, accumulates with repeated training. This is consistent with previous findings by Martin et al. (1997), in cultured Aplysia neurons, which demonstrated that repeated exposure to serotonin caused an accumulation of nuclear MAPK immunoreactivity. This accumulation was necessary to overcome a "threshold" needed for downstream gene expression. This threshold phenomenon suggests that even small changes in MAPK activity above or below this value would have profound effects on memory storage. Therefore, immediate infusion of PD098059 after training may decrease MAPK activity below this threshold value, resulting in insufficient downstream gene induction and attenuation of longterm memory. In addition, the half-life of active MAPK inside the nucleus of hippocampal neurons may be short, which would require constant rephosphorylation by MEK to have the needed cumulative effect. Similarly, the pretraining infusion of PD098059, even if the drug was only effective through part of the training protocol, would affect accumulation of activated MAPK sufficiently to impair gene expression and long-term memory formation.

Studies of MAPK phosphorylation in Hermissenda photoreceptors after classical conditioning experiments in Aplysia sensorymotor neuron cocultures, odor learning, and LTP in hippocampal slices have suggested that activation of the MAPK cascade is necessary for long-term plasticity (English and Sweatt, 1997; Martin et al., 1997; Berman et al., 1998; Crow et al., 1998). Recently, Atkins et al. (1998), using a fear-conditioning paradigm, reported that MAPK is required for associative learning. Both the behavioral protocols used in their study (contextual and cued-contextual conditioning) caused robust increases in MAPK phosphorylation in the hippocampus (shown by Western blotting of homogenized tissue). However, their protocol for contextual conditioning (where no tone was present during training, also called foreground conditioning) has been shown to be independent of hippocampal function (Phillips and LeDoux, 1994). Therefore, the increases in MAPK phosphorylation they observed may not be learning-related because it occurred in both hippocampal-dependent and hippocampal-independent tasks.

Our experiments show that MAPK-mediated changes occurring in the CA1/CA2 subfield of the dorsal hippocampus of a behaving animal are critical for long-term spatial memory formation and provide a clear launching point for further investigation into the molecular and genomic cascades involved in long-term memory. It has been reported that CREB "knock-outs", as well as rats treated with antisense oligonucleotides to downregulate CREB in the dorsal hippocampus, have impaired long-term spatial memory (Bourtchuladze et al., 1994; Guzowski and McGaugh, 1997). Several protein kinases (PKA, Akt, CaMK IV, 
MAPK via $90^{\text {rsk }}$ ) have been shown to be able to activate CREB via phosphorylation of $\operatorname{ser}^{133}$ (Yamamoto et al., 1988; Sun et al., 1994; Bito et al., 1996; Xing et al., 1996). Thus, it is possible that the activation of MAPK we observed after behavioral training may mediate its effects via CREB activation and the expression of CRE sequence-containing genes. Alternatively, MAPK can induce expression of downstream genes necessary for long-term memory, independent of CREB by phosphorylating other transcription factors such as Elk-1 (Whitmarsh and Davis, 1996). Future experiments will help distinguish between these possibilities.

\section{REFERENCES}

Abel T, Martin KC, Bartsch D, Kandel ER (1998) Memory suppressor genes: inhibitory constraints on the storage of long-term memory. Science 279:338-341.

Alberini CM, Ghirardi M, Huang YY, Nguyen PV, Kandel ER (1995) A molecular switch for the consolidation of long-term memory: cAMPinducible gene expression. Ann NY Acad Sci 758:261-286.

Alessi DR, Cuenda A, Cohen P, Dudley DT, Saltiel AR (1995) PD 098059 is a specific inhibitor of the activation of mitogen-activated protein kinase kinase in vitro and in vivo. J Biol Chem 270:27489-27494.

Amaral DG, Witter MP (1995) Hippocampal formation. In: The rat nervous system, Ed 2, pp 443-493. San Diego: Academic.

Atkins CM, Selcher JC, Petraitis JJ, Trzaskos JM, Sweatt JD (1998) The MAPK cascade is required for mammalian associative learning. Nat Neurosci 1:602-609.

Barnes CA, Jung MW, McNaughton BL, Korol DL, Andreasson K, Worley PF (1994) LTP saturation and spatial learning disruption: effects of task variables and saturation levels. J Neurosci 14:5793-5806.

Berman DE, Hazvi S, Rosenblum K, Seger R, Dudai Y (1998) Specific and differential activation of mitogen-activated protein kinase cascades by unfamiliar taste in the insular cortex of the behaving rat. J Neurosci 18:10037-10044.

Bito H, Deisseroth K, Tsien RW (1996) CREB phosphorylation and dephosphorylation: a $\mathrm{Ca}(2+)$ - and stimulus duration-dependent switch for hippocampal gene expression. Cell 87:1203-1214.

Bourtchuladze R, Frenguelli B, Blendy J, Cioffi D, Schutz G, Silva AJ (1994) Deficient long-term memory in mice with a targeted mutation of the cAMP-responsive element-binding protein. Cell 79:59-68.

Bunsey M, Eichenbaum H (1996) Conservation of hippocampal memory function in rats and humans. Nature 379:255-257.

Chen C, Tonegawa S (1997) Molecular genetic analysis of synaptic plasticity, activity-dependent neural development, learning, and memory in the mammalian brain. Annu Rev Neurosci 20:157-184.

Colombo M, Fernandez T, Nakamura K, Gross CG (1998) Functional differentiation along the anterior-posterior axis of the hippocampus in monkeys. J Neurophysiol 80:1002-1005.

Crow T, Xue-Bian JJ, Siddiqi V, Kang Y, Neary JT (1998) Phosphorylation of mitogen-activated protein kinase by one-trial and multi-trial classical conditioning. J Neurosci 18:3480-3487.

Davis HP, Squire LR (1984) Protein synthesis and memory: a review. Psychol Bull 96:518-559.

Dixon CE, Hamm RJ, Taft WC, Hayes RL (1994) Increased anticholinergic sensitivity following closed skull impact and controlled cortical impact traumatic brain injury in the rat. J Neurotrauma 11:275-287.

English JD, Sweatt JD (1997) A requirement for the mitogen-activated protein kinase cascade in hippocampal long term potentiation. J Biol Chem 272:19103-19106.

Goelet P, Castellucci VF, Schacher S, Kandel ER (1986) The long and the short of long-term memory-a molecular framework. Nature 322:419-422.

Guzowski JF, McGaugh JL (1997) Antisense oligodeoxynucleotidemediated disruption of hippocampal cAMP response element binding protein levels impairs consolidation of memory for water maze training. Proc Natl Acad Sci USA 94:2693-2698.

Hagiwara M, Brindle P, Harootunian A, Armstrong R, Rivier J, Vale W, Tsien R, Montminy MR (1993) Coupling of hormonal stimula- tion and transcription via the cyclic AMP-responsive factor CREB is rate limited by nuclear entry of protein kinase A. Mol Cell Biol 13:4852-4859.

Huang YY, Kandel ER, Varshavsky L, Brandon EP, Qi M, Idzerda RL, McKnight GS, Bourtchuladze R (1995) A genetic test of the effects of mutations in PKA on mossy fiber LTP and its relation to spatial and contextual learning. Cell 83:1211-1222.

Jung MW, Wiener SI, McNaughton BL (1994) Comparison of spatial firing characteristics of units in dorsal and ventral hippocampus of the rat. J Neurosci 14:7347-7356.

Kim JJ, Fanselow MS (1992) Modality-specific retrograde amnesia of fear. Science 256:675-677.

Klann E, Chen SJ, Sweatt JD (1993) Mechanism of protein kinase C activation during the induction and maintenance of long-term potentiation probed using a selective peptide substrate [see comments]. Proc Natl Acad Sci USA 90:8337-8341.

Kornhauser JM, Greenberg ME (1997) A kinase to remember: dual roles for MAP kinase in long-term memory [comment]. Neuron 18:839-842.

Lanahan A, Lyford G, Stevenson GS, Worley PF, Barnes CA (1997) Selective alteration of long-term potentiation-induced transcriptional response in hippocampus of aged, memory-impaired rats. J Neurosci 17:2876-2885.

Marshall CJ (1995) Specificity of receptor tyrosine kinase signaling: transient versus sustained extracellular signal-regulated kinase activation. Cell 80:179-185.

Martin KC, Michael D, Rose JC, Barad M, Casadio A, Zhu H, Kandel ER (1997) MAP kinase translocates into the nucleus of the presynaptic cell and is required for long-term facilitation in Aplysia. Neuron 18:899-912.

Meunier M, Bachevalier J, Mishkin M, Murray EA (1993) Effects on visual recognition of combined and separate ablations of the entorhinal and perirhinal cortex in rhesus monkeys. J Neurosci 13:5418-5432.

Milner B, Squire LR, Kandel ER (1998) 1998 Cognitive neuroscience and the study of memory Neuron 3:445-468.

Moore AN, Waxham MN, Dash PK (1996) Neuronal activity increases the phosphorylation of the transcription factor cAMP response element-binding protein (CREB) in rat hippocampus and cortex. J Biol Chem 271:14214-14220.

Morris RG, Garrud P, Rawlins JN, O'Keefe J (1982) Place navigation impaired in rats with hippocampal lesions. Nature 297:681-683.

Morris RG, Anderson E, Lynch GS, Baudry M (1986) Selective impairment of learning and blockade of long-term potentiation by an $N$-methyl-D-aspartate receptor antagonist, AP5. Nature 319:774-776.

Moser E, Moser MB, Andersen P (1993) Spatial learning impairment parallels the magnitude of dorsal hippocampal lesions, but is hardly present following ventral lesions. J Neurosci 13:3916-3925.

Moser MB, Moser EI, Forrest E, Andersen P, Morris RG (1995) Spatial learning with a minislab in the dorsal hippocampus. Proc Natl Acad Sci USA 92:9697-9701.

Nicoll RA, Malenka RC (1995) Contrasting properties of two forms of long-term potentiation in the hippocampus. Nature 377:115-118.

Nosten-Bertrand M, Errington ML, Murphy KP, Tokugawa Y, Barboni E, Kozlova E, Michalovich D, Morris RG, Silver J, Stewart CL, Bliss TV, Morris RJ (1996) Normal spatial learning despite regional inhibition of LTP in mice lacking Thy-1. Nature 379:826-829.

O’Dell TJ, Grant SG, Karl K, Soriano PM, Kandel ER (1992) Pharmacological and genetic approaches to the analysis of tyrosine kinase function in long-term potentiation. Cold Spring Harb Symp Quant Biol 57:517-526.

Phillips RG, LeDoux JE (1994) Lesions of the dorsal hippocampal formation interfere with background but not foreground contextual fear conditioning. Learn Mem 1:34-44.

Roberson ED, Sweatt JD (1996) Transient activation of cyclic AMPdependent protein kinase during hippocampal long-term potentiation. J Biol Chem 271:30436-30441.

Sacktor TC, Osten P, Valsamis H, Jiang X, Naik MU, Sublette E (1993) Persistent activation of the zeta isoform of protein kinase $\mathrm{C}$ in the maintenance of long-term potentiation [see comments]. Proc Natl Acad Sci USA 90:8342-8346.

Scoville WB, Milner B (1957) Loss of recent memory after bilateral hippocampal lesions. J Neurol Neurosurg Psychiatry 20:11-12. 
Squire LR (1992) Memory and the hippocampus: a synthesis from findings with rats, monkeys, and humans [published erratum appears in Psychol Rev, 1992, 99:582]. Psychol Rev 99:195-231.

Sun P, Enslen H, Myung PS, Maurer RA (1994) Differential activation of CREB by $\mathrm{Ca}$ +/calmodulin-dependent protein kinases type II and type IV involves phosphorylation of a site that negatively regulates activity. Genes Dev 8:2527-2539.

Suzuki WA, Zola-Morgan S, Squire LR, Amaral DG (1993) Lesions of the perirhinal and parahippocampal cortices in the monkey produce long-lasting memory impairment in the visual and tactual modalities. J Neurosci 13:2430-2451.

Tsien JZ, Huerta PT, Tonegawa S (1996) The essential role of hippocampal CA1 NMDA receptor-dependent synaptic plasticity in spatial memory [see comments]. Cell 87:1327-1338.

Whitmarsh AJ, Davis RJ (1996) Transcription factor AP-1 regulation by mitogen-activated protein kinase signal transduction pathways. J Mol Med 74:589-607.

Xing J, Ginty DD, Greenberg ME (1996) Coupling of the RAS-MAPK pathway to gene activation by RSK2, a growth factor-regulated CREB kinase. Science 273:959-963.

Yamamoto KK, Gonzalez GA, Biggs WH, Montminy MR (1988) Phosphorylation-induced binding and transcriptional efficacy of nuclear factor CREB. Nature 334:494-498.

Zola-Morgan SM, Squire LR (1990) The primate hippocampal formation: evidence for a time-limited role in memory storage. Science 250:288-290.

Zola-Morgan S, Squire LR, Amaral D (1986) Human amnesia and the medial temporal region: enduring memory impairment following a bilateral lesion limited to field CA1 of the hippocampus. J Neurosci 6:2950-2967. 